\title{
Randomised controlled trial of polysomnographic titration of noninvasive ventilation
}

\author{
Liam M. Hannan ${ }^{1,2,3}$, Linda Rautela ${ }^{1,2,4}$, David J. Berlowitz ${ }^{1,2,4}$ \\ Christine F. McDonald ${ }^{1,2,3}$, Jennifer M. Cori ${ }^{1}$, Nicole Sheers (iD) $1,2,4$, \\ Caroline Chao ${ }^{1,2,4}$, Fergal J. O’Donoghue ${ }^{1,2,3}$ and Mark E. Howard (i] ${ }^{1,2,3}$ \\ Affiliations: ${ }^{1}$ Institute for Breathing and Sleep, Austin Health, Melbourne, Australia. ${ }^{2}$ Dept of Respiratory and \\ Sleep Medicine, Austin Health, Melbourne, Australia. ${ }^{3}$ Dept of Medicine, Dentistry and Health Sciences, University \\ of Melbourne, Melbourne, Australia. ${ }^{4}$ Dept of Physiotherapy, University of Melbourne, Melbourne, Australia.
}

Correspondence: Liam M. Hannan, Dept of Respiratory and Sleep Medicine, Austin Health, P0 Box 5555, Heidelberg, Victoria 3084, Australia. E-mail: liam.hannandaustin.org.au

$@$ ERSpublications

In a cohort comprised mostly of individuals with neuromuscular disorders, nocturnal noninvasive ventilation was associated with less patient-ventilator asynchrony and adherence was better when treatment was titrated during polysomnography http://ow.ly/xibR30nY8c7

Cite this article as: Hannan LM, Rautela L, Berlowitz DJ, et al. Randomised controlled trial of polysomnographic titration of noninvasive ventilation. Eur Respir J 2019; 53: 1802118 [https://doi.org/ 10.1183/13993003.02118-2018].

ABSTRACT Noninvasive ventilation (NIV) settings determined during wakefulness may produce patient-ventilator asynchrony (PVA) during sleep, causing sleep disruption and limiting tolerance. This study investigated whether NIV titrated with polysomnography (PSG) is associated with less PVA and sleep disruption than therapy titrated during daytime alone.

Treatment-naive individuals referred for NIV were randomised to control (daytime titration followed by sham polysomnographic titration) or PSG (daytime titration followed by polysomnographic titration) groups. Primary outcomes were PVA and arousal indices on PSG at 10 weeks. Secondary outcomes included adherence, gas exchange, symptoms and health-related quality of life (HRQoL).

In total, 60 participants were randomised. Most (88.3\%) had a neuromuscular disorder and respiratory muscle weakness but minor derangements in daytime arterial blood gases. PVA events were less frequent in those undergoing polysomnographic titration (median (interquartile range (IQR)): PSG 25.7 (1268) events $\cdot \mathrm{h}^{-1}$ versus control $41.0(28-182)$ events $\left.\cdot \mathrm{h}^{-1} ; \mathrm{p}=0.046\right)$, but arousals were not significantly different (median (IQR): PSG 11.4 (9-19) arousals $\cdot \mathrm{h}^{-1}$ versus control $14.6(11-19)$ arousals $\left.\cdot \mathrm{h}^{-1} ; \mathrm{p}=0.258\right)$. Overall adherence was not different except in those with poor early adherence $\left(<4 \mathrm{~h} \cdot \mathrm{day}^{-1}\right)$ who increased their use after polysomnographic titration (mean difference: PSG 95 (95\% CI 29-161) min.day ${ }^{-1}$ versus control -23 (95\% CI -86-39) min.day $\left.{ }^{-1} ; \mathrm{p}=0.01\right)$. Arterial carbon dioxide tension, somnolence and sleep quality improved in both groups. There were no differences in nocturnal gas exchange or overall measures of HRQoL.

NIV titrated with PSG is associated with less PVA but not less sleep disruption when compared with therapy titrated during daytime alone.

This article has supplementary material available from erj.ersjournals.com

Received: Nov 062018 | Accepted after revision: March 032019

This study was registered prospectively with the Australian New Zealand Clinical Trials Registry with identifier number 365405. Individual participant data that underlie the results reported in this article will be available for sharing after deidentification (text, tables, figures and appendices). The study protocol will also be available. These data will be available beginning 12 months and ending 36 months following article publication to researchers who provide a methodologically sound proposal and with approval of the Research Ethics Board who originally approved the study. Proposals should be directed to liam.hannan@austin.org.au. To gain access, data requestors will need to sign a data access agreement.

Copyright @ERS 2019 


\section{Introduction}

Long-term noninvasive ventilation (NIV) is an effective treatment for many individuals with or at risk of chronic hypercapnic respiratory failure [1-5]. Despite this, consensus on how best to implement NIV for treatment-naive users is lacking $[1,5,6]$. A variety of implementation methods have been reported, ranging from inpatient hospital assessments to ambulatory or home-based models [1, 7-11]. Reports have also described varying combinations of clinical assessments and other measures that may assist with treatment initiation and monitoring. These include daytime arterial blood gases, nocturnal oximetry, capnography and device-derived data, as well as polygraphy or polysomnography (PSG) [1, 12]. Some of the variation in practice may be due to differences in healthcare systems (particularly funding) as well as differences in access to NIV [5]. There are very few studies comparing different techniques and therefore objective evidence is not a major driver of practice.

Polygraphy and PSG monitoring of NIV are unique in that they allow observation of patient-ventilator interaction during nocturnal use and (in the case of PSG) an assessment of sleep quality and sleep disruption. Patient-ventilatory asynchrony (PVA) refers to a mismatch between neural (user) and mechanical (ventilator) inspiratory time [13-21]. Uncontrolled studies have demonstrated frequent PVA in those using nocturnal NIV following daytime titration [20], which improved with adjustments to settings [22]. Associations have been demonstrated between increased levels of PVA and more EEG arousals during sleep [23], less rapid eye movement sleep and lower sleep efficiency [22], worse nocturnal gas exchange [20], and reduced NIV tolerance [24]. Polysomnographic titration of NIV has therefore been recommended [25], but no controlled trials of this technique have been performed.

The aim of this study was to determine whether the routine use of polysomnographic titration of NIV is associated with significant physiological and clinical benefits for treatment-naive users. The hypotheses tested were that NIV titrated with PSG would be associated with lower PVA and EEG arousal indices during sleep than NIV titrated during daytime titration alone. It was also hypothesised that NIV titrated with PSG would be associated with greater adherence to therapy and larger improvements in gas exchange, symptoms and health-related quality of life (HRQoL).

\section{Methods}

Study design and participants

The study was a single-centre, blinded, parallel group, randomised controlled trial approved by the Research Ethics Board of Austin Health (Melbourne, Australia) and registered prospectively with the Australian New Zealand Clinical Trials Registry (identifier 365405). All subjects provided informed consent. Consecutive, medically stable adults with, or at risk of, chronic hypercapnic respiratory failure who were referred for long-term domiciliary NIV at a state-wide home mechanical ventilation (HMV) service in Melbourne were invited to participate. The decision to initiate long-term NIV was made by the referring specialist clinician in accordance with published guidelines [6, 26-28]. Exclusion criteria included current inpatient hospitalisation, hypoventilation attributable to sedative/respiratory depressant medications, use of assisted ventilation in any form (but not including continuous positive airway pressure (CPAP)) for $>1$ month in the previous 3 months, lack of proficiency in English, inability to provide informed consent or documented history of NIV intolerance.

\section{Procedures}

Baseline

At enrolment, all participants underwent arterial blood gas measurements on room air and completed questionnaires evaluating somnolence (Epworth Sleepiness Scale [29] and Karolinska Sleepiness Scale [30]), sleep quality (Pittsburgh Sleep Quality Index [31]), dyspnoea (Modified Borg Dyspnoea Scale) and fatigue (Fatigue Severity Scale [32]). Disease-specific (Severe Respiratory Insufficiency Questionnaire (SRI) [33]) and generic (Assessment of Quality of Life, 8-Dimension [34]) HRQoL instrument responses were also collected.

\section{Daytime titration}

All participants then underwent a standardised daytime titration of NIV performed by one of two respiratory physiotherapists over at least $4 \mathrm{~h}$ within a modified sleep laboratory located on a respiratory inpatient ward [10]. This procedure was consistent with clinical practice immediately prior to study initiation, and involved interface selection, initial acclimatisation to NIV and subsequent titration of NIV settings to optimise comfort, leak, patient-ventilator synchronisation, minute ventilation and arterial oxygen saturation measured by pulse oximetry. Further details on the daytime titration are provided in the supplementary material. 
Following the daytime titration, a trained nurse provided a standardised education session that included information on the care and use of equipment. Participants returned home on the same day and were provided with access to 24-h telephone support and a nursing outreach service.

\section{Intervention}

Following an acclimatisation period of 2-3 weeks (figure 1 and supplementary material), participants were randomly allocated to either polysomnographic titration (PSG group) or sham polysomnographic titration (control group). Those in the PSG group underwent an attended in-laboratory overnight PSG (E-series PSG; Compumedics, Abbotsford, Australia). In addition to standard recorded parameters, other measures included surface diaphragm electromyography, mask pressure, and calibrated ventilator airflow and leak signals obtained using a proprietary device (ResMed, San Diego, CA, USA). Transcutaneous carbon dioxide tension was measured continuously (TCM-4; Radiometer, Copenhagen, Denmark), and corrected for drift and offset using arterial carbon dioxide tension $\left(\mathrm{PaCO}_{2}\right)$ [35]. Sleep scientists experienced in polysomnographic titration adjusted the NIV settings (and interface) according to a standardised approach (supplementary material).

The control group underwent in-laboratory PSG using identical procedures to the PSG group, but without setting or interface adjustments. Attending sleep scientists did not reveal group allocation to participants in either group. For the control group, the sleep scientist would only provide assistance to participants on request and did not intervene to correct ventilator issues or respond to physiological derangements that were observed during the recordings.

Following the intervention, polysomnographic recordings were reviewed by two of four research team members (M.E.H., F.J.O’D., L.R. and N.S.). Recommended settings were obtained by consensus for the PSG group and written on a standardised form. Settings were also provided for the control group to avoid revealing group allocation. A blinded member of the research team (L.M.H.) then programmed settings for both groups onto data cards, SD cards or USB sticks and these were sent to participants with instructions on how to update their devices.

At study conclusion, participants completed symptom and HRQoL questionnaires, repeat daytime arterial blood gas assessments, and undertook in-laboratory PSG with their current NIV settings and interface. No

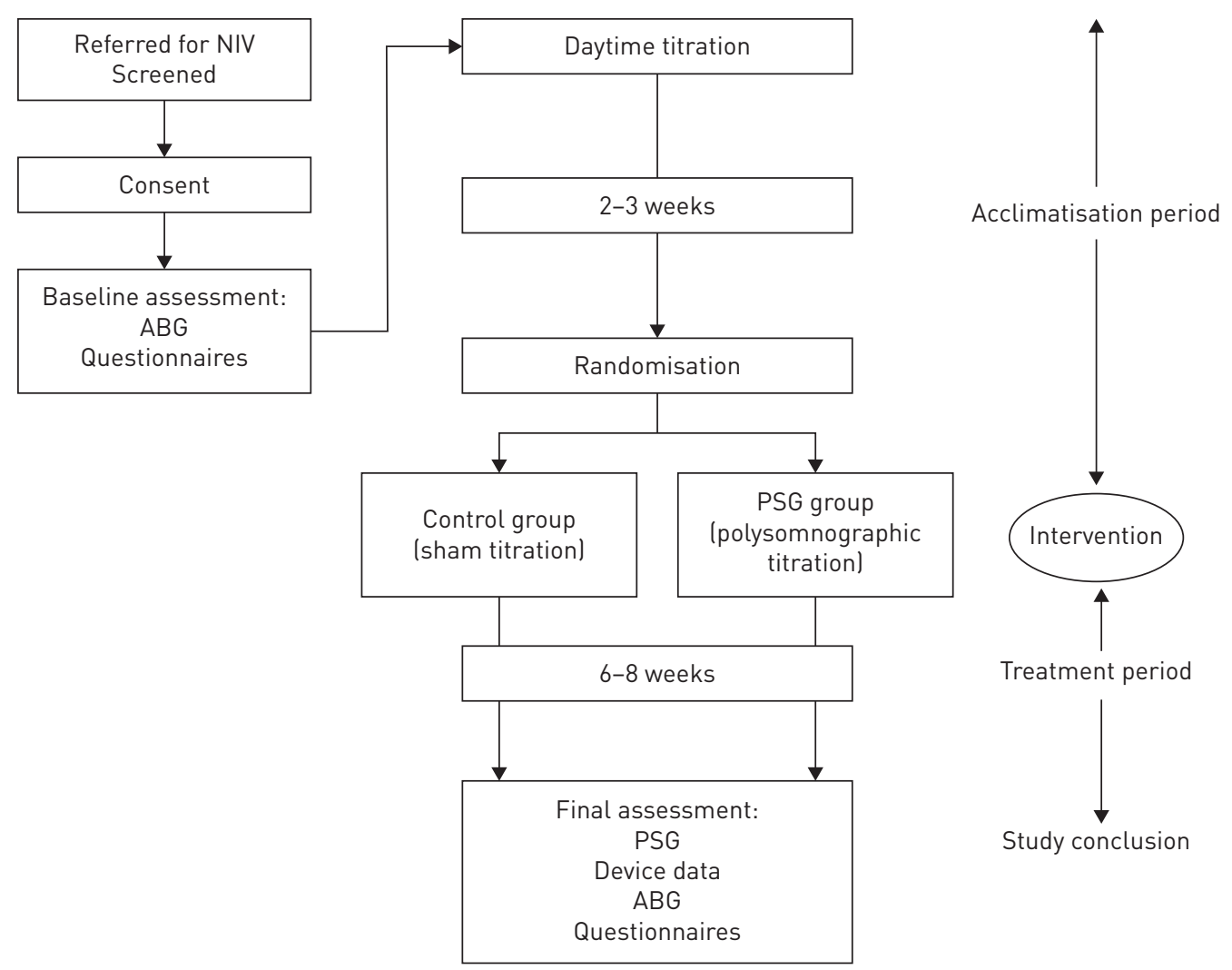

FIGURE 1 Study procedure flowchart. NIV: noninvasive ventilation; ABG: arterial blood gas; PSG: polysomnography. 
NIV alterations were made during this second overnight recording. Adherence data were downloaded from NIV devices.

\section{Randomisation and blinding}

Simple randomisation using blocks of 10 participants was performed by a member of the research team not involved in the participants' clinical management or identified outcome measure analyses (D.J.B.). Allocation concealment was ensured through the use of sealed opaque envelopes. Randomisation was performed during the acclimatisation period, but after the daytime titration process had been completed.

The attending sleep scientist and the team evaluating the PSG recordings were unblinded to group allocation by necessity. Once unblinded, these individuals were not involved in further data collection or analyses. Participants were blinded to group allocation as were those individuals involved in the collection of outcome measures, the staging/scoring polysomnographic data, and those supporting and managing participants.

\section{Outcome measures}

The co-primary outcomes were measures of synchronisation (the PVA index) and sleep disruption (the arousal index) on the final overnight PSG performed at study conclusion.

The PVA index was calculated as the number of asynchrony events per hour of sleep (see supplementary material for details) $[21,22,36]$. Indices for each of the scored events (ineffective efforts, double-trigger events and multiple-trigger events) were also calculated. A single scorer (L.M.H.) identified PVA events by inspecting each breath within each 30-s epoch on de-identified polysomnographic recordings examined without access to EEG or other sleep staging data.

The arousal index was defined as the number of EEG arousals observed per hour of sleep. All polysomnographic recordings were staged and scored according to standard criteria by a single experienced sleep scientist unaware of the study hypothesis [37].

Secondary outcome measures included device-derived adherence data, objective sleep quality, gas exchange (daytime and nocturnal) and patient-reported outcomes.

Changes in adherence were compared between the acclimatisation and treatment periods (figure 1). Nonadherence was defined as average daily use of $<4 \mathrm{~h}[38,39]$. Objective sleep quality and nocturnal gas exchange measures were obtained from the PSG performed at study conclusion [37].

Changes in patient-reported outcome measures were determined from baseline (prior to NIV therapy) and study conclusion. Contacts with healthcare workers, emergency department presentations or contacts with primary care physicians were collected from the HMV service records and from self-report.

\section{Sample size calculation}

Based on observational data from Adler et al. [18] and FAnfulla et al. [22], it was estimated that a sample size of $n=40$ (20 per group) would provide $80 \%$ power at an $\alpha$-level of 0.05 to detect a $50 \%$ difference in both the PVA and arousal indices, and allow up to $15 \%$ dropouts. Planned subgroup analyses included individuals with motor neuron disease (MND), individuals with obesity hypoventilation syndrome (OHS), individuals with MND and severe bulbar dysfunction, and individuals with nonadherence during the acclimatisation period. These subgroup analyses increased the projected sample size to $n=110$ ( 55 per group).

\section{Statistical evaluation}

Statistical calculations were performed using SPSS version 21 (IBM, Armonk, NY, USA) and Excel 2007 (Microsoft, Redmond, WA, USA). Graphs were produced using Prism version 6 (GraphPad, La Jolla, CA, USA). Results are presented as mean with standard deviation or median (interquartile range (IQR)) as appropriate. Between-group comparisons were performed using Pearson's Chi-squared test for proportions, the t-test for the comparison of means and the Mann-Whitney U-test for nonnormally distributed data. A p-value $<0.05$ was considered statistically significant.

\section{Results}

During the recruitment period (December 2013 to December 2015), 313 individuals commenced HMV (figure 2). Of these, 52.1\% $(n=163)$ were screened and of these, $31.9 \%(n=52)$ were excluded. Most of the 51 potential participants who declined to participate cited unwillingness to attend for additional overnight monitoring or difficulty with transport.

An unanticipated fall in referrals for individuals with OHS resulted in insufficient recruitment to achieve the target sample size within a reasonable timeframe. The study investigators therefore concluded recruitment after $\mathrm{n}=60$ participants were accrued. 


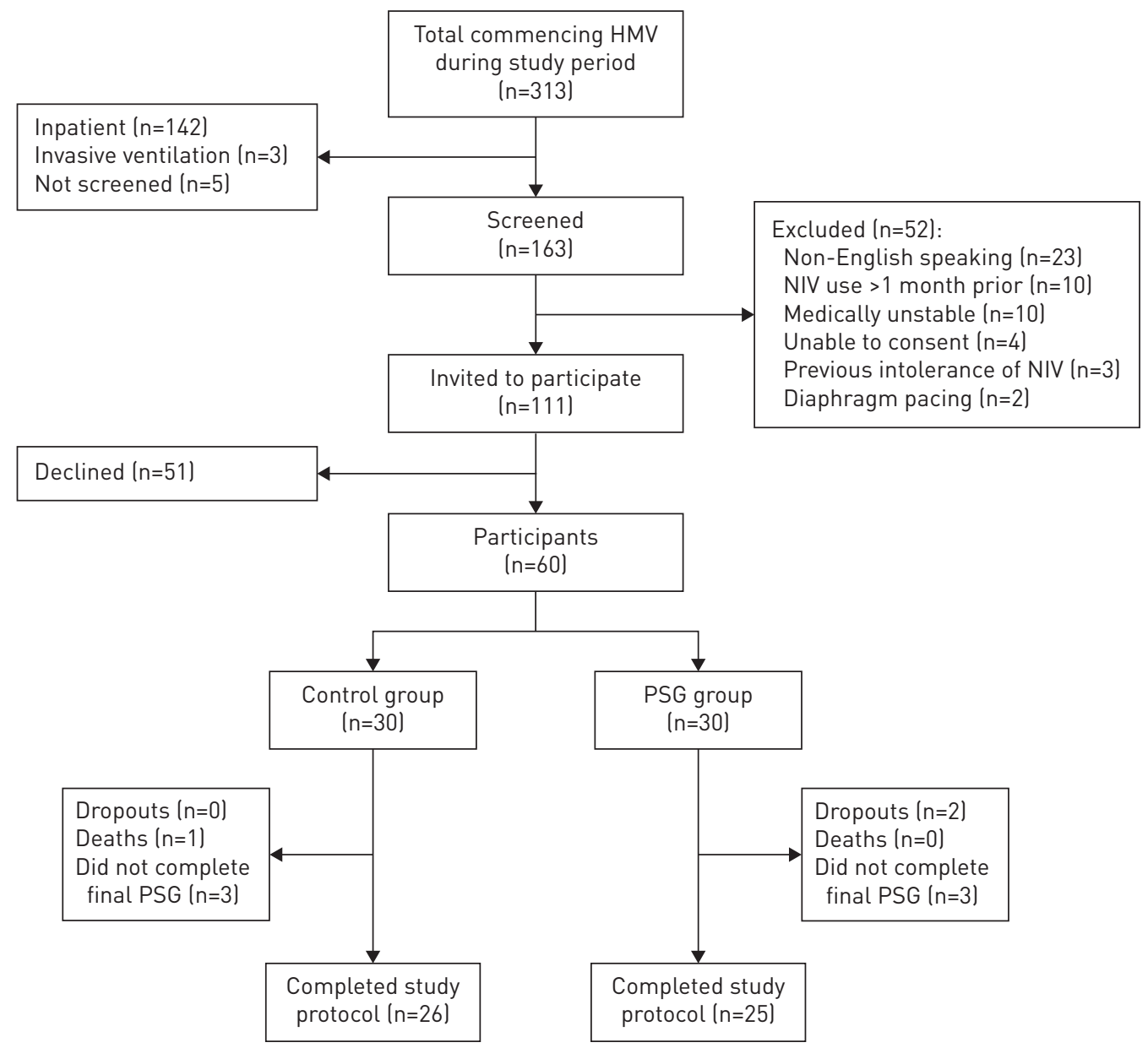

FIGURE 2 CONSORT diagram. HMV: home mechanical ventilation; NIV: noninvasive ventilation; PSG: polysomnography.

Both groups were well matched at baseline for age, sex, body mass index, diagnosis and respiratory function (table 1). Most participants had neuromuscular disease (most frequently MND) with evidence of respiratory muscle weakness. The median (IQR) daytime $\mathrm{PaCO}_{2}$ was within normal limits for both groups (control 43 (39-48) $\mathrm{mmHg}$ and PSG $45(38-51) \mathrm{mmHg}$ ). Most participants were naive to all forms of positive pressure therapy (table 1).

\section{Acclimatisation period}

The mean \pm SD daily use in both groups was $>4 \mathrm{~h}$ (control $305 \pm 185 \mathrm{~min} \cdot \mathrm{day}^{-1}$ and PSG $291 \pm 197 \mathrm{~min} \cdot \mathrm{day}^{-1}$ ) during the acclimatisation period (supplementary table E2), but nonadherence (defined using the 4-h threshold) was common (control $n=9(30 \%)$ and PSG $n=12(40 \%)$ ) (supplementary table E2). The initial settings determined during the daytime titration were similar between groups (supplementary table E1) and all except two participants used an oronasal mask. Those with previous experience with CPAP were not more or less adherent than those who were naive to noninvasive interfaces (supplementary table E2).

\section{Polysomnographic titration and alterations to settings}

Polysomnographic titration resulted in adjustment in at least one ventilator parameter in $96.7 \%(\mathrm{n}=29)$ of the PSG group. The most frequently altered parameters were the expiratory pressure and pressure support (i.e. inspiratory minus expiratory pressure), which tended to increase when compared with the original settings (supplementary table E3).

\section{Primary outcomes}

The median (IQR) PVA index was significantly higher in the control group (control 41.0 (28-182) events.h ${ }^{-1}$ versus PSG 25.7 (12-68) events. $\left.\mathrm{h}^{-1} ; \mathrm{p}=0.046\right)$ (table 2 and figure $3 \mathrm{a}$ ) and primarily attributable to a higher frequency of ineffective efforts. 
TABLE 1 Baseline characteristics of the study population

\begin{tabular}{|c|c|c|}
\hline & Control group & PSG group \\
\hline Subjects & 30 & 30 \\
\hline Age years & $60 \pm 9$ & $63 \pm 12$ \\
\hline Male/female & $23 / 7$ & $20 / 10$ \\
\hline $\mathrm{BMI} \mathrm{kg} \cdot \mathrm{m}^{-2}$ & $29.5 \pm 11.5$ & $27.3 \pm 6.6$ \\
\hline Comorbidities & $1.0 \pm 1.4$ & $1.37 \pm 1.4$ \\
\hline \multicolumn{3}{|l|}{ Diagnosis } \\
\hline MND & 18 & 22 \\
\hline ALSFRS-R score & $29.3 \pm 7$ & $28.0 \pm 7$ \\
\hline Bulbar (ALSFRS) score & $8.7 \pm 3$ & $7.1 \pm 4$ \\
\hline Riluzole at enrolment \% & 56 & 73 \\
\hline NMD & 7 & 6 \\
\hline RTD & 0 & 2 \\
\hline $\mathrm{OHS}$ & 3 & 0 \\
\hline COPD/OSA & 2 & 0 \\
\hline FVC \% pred & $55 \pm 14$ & $54 \pm 15$ \\
\hline MIP $\mathrm{cmH}_{2} \mathrm{O}$ & $30(23-54)$ & $28(22-33)$ \\
\hline Daytime $\mathrm{PaCO}_{2} \mathrm{mmHg}$ & $43(39-48)$ & $45(38-51)$ \\
\hline Previous CPAP ${ }^{\#}$ & 36.7 & 20.0 \\
\hline \multicolumn{3}{|c|}{$\begin{array}{l}\text { Data are presented as } \mathrm{n} \text {, mean } \pm \text { SD or median (interquartile range). BMI: body mass index; MND: motor } \\
\text { neuron disease; ALSFRS: Amyotrophic Lateral Sclerosis Functional Rating Scale; NMD: neuromuscular } \\
\text { disorder; RTD: restrictive thoracic disorder; OHS: obesity hypoventilation syndrome; COPD/OSA: chronic } \\
\text { obstructive pulmonary disease with obstructive sleep apnoea; FVC: forced vital capacity; MIP: maximum } \\
\text { inspiratory pressure; } P_{a C_{2}} \text { : arterial carbon dioxide tension; CPAP: continuous positive airway pressure. } \\
\# \text { : CPAP previously prescribed, used or evaluated in a laboratory or inpatient setting. }\end{array}$} \\
\hline
\end{tabular}

There was no significant difference between the two groups in the median (IQR) arousal index (control 14.6 (11-19) arousals $\cdot \mathrm{h}^{-1}$ versus PSG $11.4(9-19)$ arousals $\left.^{-1} \mathrm{~h}^{-1} ; \mathrm{p}=0.258\right)$ (figure $3 \mathrm{~b}$ and supplementary table E6).

\section{Secondary outcomes}

Change in adherence

The PSG group significantly increased their average daily use of NIV overall (figure 4), with the improvement significant in the between-group comparison in those who were nonadherent during acclimatisation (95 versus $-23 \mathrm{~min} ; \mathrm{p}<0.01$ ). No change in adherence was observed overall or in nonadherent participants allocated to the control group (table 3 and figure 4).

Gas exchange and sleep quality

Daytime $\mathrm{PaCO}_{2}$ fell by a similar magnitude in both groups following the introduction of nocturnal NIV (control ( $\mathrm{n}=25) 3.25 \mathrm{mmHg}$ versus PSG $(\mathrm{n}=28) 3.07 \mathrm{mmHg}$; $=0.92)$ and no difference was demonstrated in nocturnal measures of gas exchange (supplementary table E7). Objective measures of sleep quality were also not significantly different (supplementary table E6).

TABLE 2 Patient-ventilator asynchrony (PVA) events (by type) per hour of total sleep time (TST): polysomnography (PSG) group compared with control group

\begin{tabular}{lccc} 
& Control group & PSG group & p-value $^{\#}$ \\
\hline Subjects & $25^{\text {q }}$ & 25 & \\
PVA index total events $\cdot h^{-1}$ TST & $41.0(28-182)$ & $25.7(12-68)$ & 0.046 \\
Ineffective efforts events $\cdot \mathbf{h}^{-1}$ TST & $26.4(15-107)$ & $13.6(5-37)$ & 0.040 \\
Double-trigger events $\mathbf{h}^{-1}$ TST & $5.5(3-33)$ & $5.6(2-11)$ & 0.594 \\
Multiple-trigger events $\cdot \mathbf{h}^{-1}$ TST & $0.5(0-4)$ & $0.5(0-2)$ & 0.576 \\
\hline
\end{tabular}

Data are presented as $\mathrm{n}$ or median (interquartile range), unless otherwise stated. \#: $\mathrm{p}$-value from independent samples Mann-Whitney U-test; " : one participant in the control group had malfunction in mask pressure trace and therefore no PVA events were obtained. 

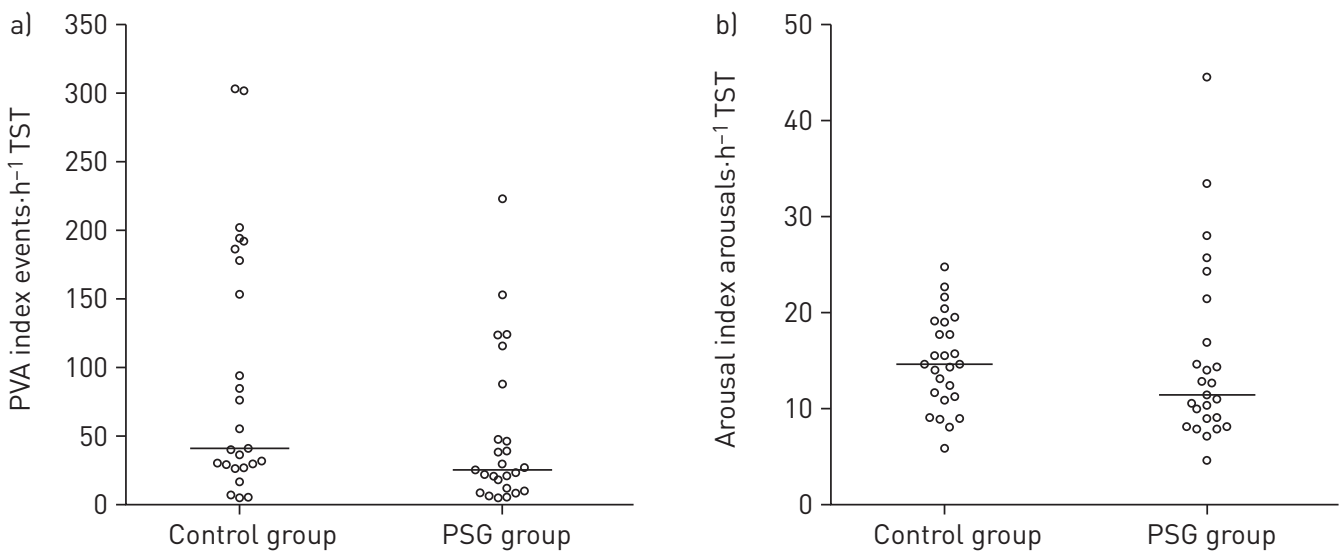

FIGURE 3 a) Patient-ventilator asynchrony (PVA) index and b) arousal index during polysomnography (PSG) at study conclusion: PSG group compared with control group. TST: total sleep time. Data are presented as individual data points; the horizontal line indicates the median value for each group.

\section{Patient-reported outcomes}

Both groups experienced significant improvements in subjective sleep quality (table 4). The PSG group demonstrated a significant, within-group mean $\pm \mathrm{SD}$ reduction in somnolence (Epworth Sleepiness Scale $-1.9 \pm 3.8 ; \mathrm{p}=0.01$ ) (table 4), but no significant between-group difference was observed. No significant changes were observed within or between groups in ratings of dyspnoea, fatigue or side-effects related to NIV. The frequency of contact with healthcare services was also not different (table 4).

\section{Health-related quality of life}

There was no overall difference in disease-specific HRQoL using the SRI Summary Scale (table 4). The PSG titration group demonstrated a significant mean \pm SD improvement in the Attendant Symptoms and Sleep domain of the SRI $(+7.9 \pm 13 ; \mathrm{p}<0.05)$ (table 4$)$ and a significant deterioration was observed in the Social Relationships domain for the control group, which was statistically significant in the between-group analysis (control $-7.6 \pm 14$ versus PSG $0.7 \pm 12 ; \mathrm{p}=0.022$ ) (table 4). The generic preference-based HRQoL instrument demonstrated no significant difference between- or within-group in the index score or any of the dimension scores (supplementary table E8).

\section{Discussion}

This is the first randomised controlled trial to evaluate the influence of polysomnographic titration of NIV after daytime titration. A number of significant findings were identified. First, those who underwent polysomnographic titration of NIV had a lower PVA index compared with those undergoing only daytime
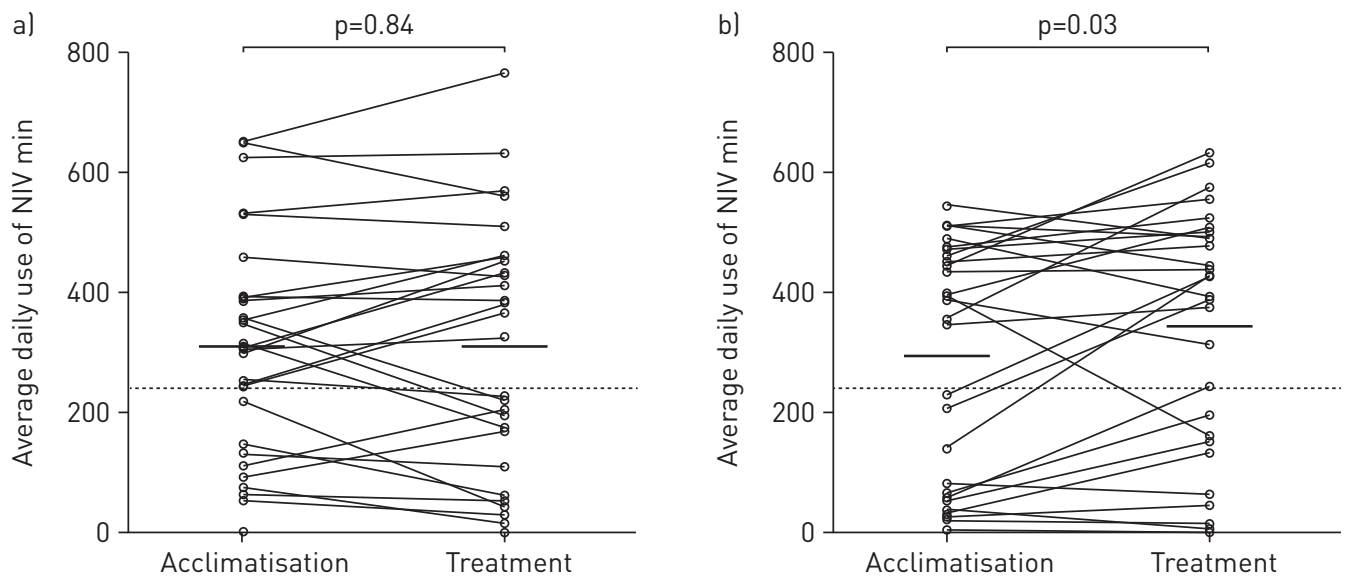

FIGURE 4 Average daily use of noninvasive ventilation (NIV) during acclimatisation and treatment periods: a) control group and b) polysomnography group. Data are presented as individual data points connected for each participant; the solid horizontal line indicates the group mean. The 4-h threshold for adherence is indicated by the dashed horizontal line. $p$-values from related samples t-test. 


\begin{tabular}{|c|c|c|c|}
\hline & Subjects $\mathbf{n}$ & Mean difference ${ }^{\#} \min (95 \% \mathrm{Cl})$ & p-value ${ }^{\pi}$ \\
\hline \multicolumn{4}{|l|}{ Overall } \\
\hline Control group & $28^{+}$ & $3.6(-33-40)$ & 0.09 \\
\hline PSG group & 28 & $51.8 *(7-96)$ & \\
\hline \multicolumn{4}{|c|}{ Adherent $(\geqslant 4 h)^{\S}$ during acclimatisation } \\
\hline Control group & 19 & $16(-31-64)$ & 0.93 \\
\hline PSG group & 16 & $19(-42-81)$ & \\
\hline \multicolumn{4}{|c|}{ Nonadherent $(<4 \mathrm{~h})^{\S}$ during acclimatisation } \\
\hline Control group & 9 & $-23(-86-39)$ & 0.01 \\
\hline PSG group & 12 & $95 *(29-161)$ & \\
\hline
\end{tabular}

PSG: polysomnography. ${ }^{\#}$ : mean difference refers to the average daily use during the treatment period minus the average daily use during the acclimatisation period; " ": between-group $p$-value from independent samples t-test; ${ }^{+}$: adherence data not available for one participant in the control group due to malfunctioning data card; ${ }^{\S}$ : adherence defined as average use $\geqslant 4 \mathrm{~h}$ per 24 -h period and nonadherence defined as average use $<4 \mathrm{~h}$ per 24 -h period. *: within-group $p<0.05$ from related samples t-test.

TABLE 4 Patient-reported outcome measures for the control and polysomnography (PSG) groups

\begin{tabular}{|c|c|c|c|c|c|}
\hline & \multicolumn{2}{|c|}{ Control group } & \multicolumn{2}{|c|}{ PSG group } & \multirow[t]{2}{*}{ p-value } \\
\hline & $\begin{array}{l}\text { Subjects } \\
n\end{array}$ & $\begin{array}{l}\text { Mean } \pm \text { sD } \\
\text { difference }\end{array}$ & $\begin{array}{c}\text { Subjects } \\
n\end{array}$ & $\begin{array}{l}\text { Mean } \pm s D \\
\text { difference }\end{array}$ & \\
\hline PSQI & 29 & $-2.1 \pm 3.9 *$ & 28 & $-1.4 \pm 3.0 *$ & 0.486 \\
\hline ESS & 29 & $-1.3 \pm 5.0$ & 28 & $-1.9 \pm 3.8^{*}$ & 0.619 \\
\hline $\mathrm{KSS}^{+}$ & 29 & $-0.4 \pm 1.7$ & 28 & $-1.1 \pm 2.5$ & 0.201 \\
\hline FSS & 29 & $-3.7 \pm 17.1$ & 28 & $-1.3 \pm 11.4$ & 0.522 \\
\hline FSS VAS & 29 & $0.5 \pm 2.7$ & 27 & $0.6 \pm 2.1$ & 0.781 \\
\hline MBDS & 28 & $0.2 \pm 2.2$ & 28 & $0.3 \pm 1.6$ & 0.836 \\
\hline SRI ${ }^{\S}$ Summary Scale & 29 & $0.3 \pm 11$ & 28 & $1.7 \pm 9$ & 0.612 \\
\hline Respiratory Complaints & 29 & $4.1 \pm 20$ & 28 & $2.4 \pm 19$ & 0.735 \\
\hline Physical Functioning & 29 & $-2.4 \pm 15$ & 28 & $0.0 \pm 18$ & 0.587 \\
\hline $\begin{array}{l}\text { Attendant Symptoms and } \\
\text { Sleep }\end{array}$ & 29 & $5.2 \pm 18$ & 28 & $7.9 \pm 13^{*}$ & 0.513 \\
\hline Social Relationships & 29 & $-7.6 \pm 14^{*}$ & 28 & $0.7 \pm 12$ & 0.022 \\
\hline Anxiety & 29 & $4.2 \pm 21$ & 28 & $3.1 \pm 18$ & 0.834 \\
\hline Psychological Wellbeing & 29 & $-1.6 \pm 13$ & 28 & $0.8 \pm 10$ & 0.432 \\
\hline \multirow[t]{2}{*}{ Social Functioning } & 29 & $0.5 \pm 16$ & 28 & $-3.0 \pm 18$ & 0.455 \\
\hline & & Mean士sd & & Mean士sd & \\
\hline SAQLI side-effects ${ }^{f}$ & 28 & $10.6 \pm 4.5$ & 28 & $11.1 \pm 4.4$ & 0.652 \\
\hline $\begin{array}{l}\text { SAQLI side-effects versus } \\
\text { benefits }\end{array}$ & 28 & $2.7 \pm 1.5$ & 28 & $2.6 \pm 1.2$ & 0.766 \\
\hline Healthcare contacts & 29 & $2.6 \pm 1.9$ & 28 & $2.7 \pm 2.9$ & 0.888 \\
\hline Database contacts & 30 & $3.2 \pm 2.6$ & 30 & $4.2 \pm 4.8$ & 0.321 \\
\hline
\end{tabular}

PSQI: Pittsburgh Sleep Quality Index; ESS: Epworth Sleepiness Scale; KSS: Karolinska Sleepiness Scale; FSS: Fatigue Severity Scale; VAS: Visual Analogue Scale; MBDS: Modified Borg Dyspnoea Score; SRI: Severe Respiratory Insufficiency Questionnaire; SAQLI: Sleep Apnoea Quality of Life Questionnaire. \#: mean difference represents the final measure (at study conclusion) minus the baseline measure (prior to commencing noninvasive ventilation) (with the exception of the FSS VAS, negative values indicate an improvement within the measure); $\eta^{\text {: }}$ : between-group $\mathrm{p}$-value from independent samples $\mathrm{t}$-test; ${ }^{+}$: responses to the KSS were not adequately matched for time of day for the majority of participants; §: domains of the SRI are scored from 0 to $100 ;{ }^{f}$ : scored as the sum of ratings (on a 7-point Likert scale) for each of the most troubling side-effects (lower scores therefore represent less troubling side-effects); \#\#: scored on a 7-point Likert scale where the middle value $(=4)$ represents a balance between side-effects and benefits (scores <4 therefore indicate that the benefits of therapy outweigh the side-effects); " self-reported contacts with healthcare workers. *: within-group $p<0.05$ from related samples t-test. 
titration, confirming findings suggested in previous, uncontrolled studies [18, 22]. Second, despite improved measures of synchronisation between the device and the user during sleep, sleep disruption (as summarised by EEG arousals) was not significantly different. A potentially important clinical effect of polysomnographic titration was identified in a pre-specified subgroup with poor early adherence to NIV. Those in the PSG group significantly increased their average daily use in contrast to those in the control group whose adherence did not change. Other short-term secondary outcomes (gas exchange, symptoms or HRQoL) did not appear to be influenced by polysomnographic titration in this stable outpatient population.

Previous observational studies by Fanfulla et al. [20,22] demonstrated that PVA events are common when NIV settings are determined in awake individuals, and that altering NIV parameters can reduce the frequency of PVA events during sleep and improve sleep quality. What remained unclear from their uncontrolled short-term physiological study (which adjusted NIV pressures by targeting reductions in transdiaphragmatic pressure swings) was how best to adjust ventilator settings in order to reduce PVA. The current study extends these findings by demonstrating an effective and clinically feasible titration method that is associated with lower levels of PVA. The influence of polysomnographic titration on synchronisation appears to be mediated primarily through a reduction in ineffective efforts, with this form of PVA previously noted to predominate [21, 36]. However, the infrequency of double- and multiple-trigger events in this study may mean it was underpowered to detect a difference in these forms of PVA. It remains uncertain whether PSG is required in order to achieve optimal titration of NIV and more limited forms of nocturnal respiratory monitoring may prove equally effective [40].

In this study, a significant effect of polysomnographic titration was identified on the average daily use of NIV in individuals with poor adherence during the acclimatisation period. The mean increase in average daily use of $95 \mathrm{~min}$ for this group was large and likely to be of clinical importance. NicKOL et al. [38] previously described a threshold of $4 \mathrm{~h}$ average daily use in individuals with neuromuscular and restrictive thoracic disorders, below which improvements in symptoms and $\mathrm{PaCO}_{2}$ would not be expected. Shifting individuals above this threshold not only suggests an important clinical effect of polysomnographic titration, but also an easily identifiable cohort of users in which to apply it. Cost-effective methods of optimising adherence to NIV will become increasingly important if the indications for this therapy are broadened, particularly in health settings where prolonged inpatient evaluation and acclimatisation is expensive or unavailable.

Despite the associations between polysomnographic titration and better synchronisation and adherence with NIV, other clinical benefits were not demonstrated in this short-term study. Improvements in symptoms were similar with both titration approaches and there were only minor differences in disease-specific HRQoL domains. It is possible that the time period (10 weeks between enrolment and study conclusion) was too short for differences in adherence to therapy to manifest as clinically important differences in symptoms and HRQoL. The clinical stability of participants at enrolment (evidenced by their outpatient status and baseline daytime $\mathrm{PaCO}_{2}$ within the normal range) may also have contributed to the lack of difference between groups. It is, however, reasonable to speculate that a titration strategy associated with a higher frequency of satisfactory adherence would result in less morbidity and mortality over time, especially in cohorts with a large proportion of individuals with progressive neuromuscular disorders $[41,42]$.

\section{Limitations}

This was a single-centre randomised controlled trial in a clinically stable population comprising primarily individuals with neuromuscular disorders and this limits the generalisability of these findings. The majority of the study population was referred for NIV due to symptoms (orthopnoea, dyspnoea and sleep fragmentation) combined with evidence of significant respiratory muscle weakness, rather than impending or unstable respiratory failure. As a result, this population may have required relatively low levels of pressure support in comparison with other long-term NIV cohorts. Ideally, including hospital inpatients and medically unstable individuals may have produced more generalisable findings; however, it was determined that there was insufficient clinical equipoise at our centre (where polysomnographic titration is routine practice) to withhold this intervention in these groups. We would hypothesise that in less stable populations (with greater derangements in sleep quality, respiratory mechanics and gas exchange) the supportive effect of polysomnographic titration on adherence and synchronisation would produce greater clinical benefits.

Changing referral patterns during the study resulted in a relative lack of individuals with OHS in comparison to the predicted population. Failing to reach the recruitment target may have contributed to the apparent lack of effect of polysomnographic titration on important clinical end-points and the potential for a type II error is acknowledged. 
Additionally, it is acknowledged that failure to perform baseline PSG (pre- and post- commencement of NIV) for both groups may limit confidence in the study findings. However, such an approach was felt to impose excessive burden on participants, many of whom were highly dependent on care. It also would have represented a significant departure from standard care at this centre. Future studies will need to carefully consider the burdens imposed to ensure participation is feasible and results are generalisable. Incorporation of home-based monitoring of physiological and ventilator data may prove useful in this regard.

\section{Conclusions}

In this single-centre study including a medically stable, outpatient cohort with predominantly neuromuscular disorders, titration of NIV using PSG was associated with better patient-ventilator synchronisation but no difference in sleep disruption. Individuals who had poor adherence to NIV during the acclimatisation period increased their average daily usage if they underwent polysomnographic titration, but other clinical benefits were not identified in this population. A potential effect on adherence is compelling, with nonadherence to NIV representing both a therapeutic challenge and an opportunity. Further research should explore the influence that titration method has on adherence and other clinically important outcome measures over a longer timeframe and include less stable individuals. A multicentre study would allow further exploration of the influence that clinician expertise and the approach to clinical titration has on the effectiveness of NIV.

Acknowledgements: The authors would like to acknowledge the considerable assistance of the administrative staff of the Victorian Respiratory Support Service (including Georgina Broumis, Ian Batchelder and Christine Stewart) and the Victorian Respiratory Support Service Outreach team.

Author contributions: L.M. Hannan was responsible for the study conception, design, data collection, analysis, coordination and preparation of the manuscript. L. Rautela, N. Sheers and C. Chao assisted with study design, data collection and analysis. J.M. Cori assisted with preparation of the manuscript. D.J. Berlowitz, C.F. McDonald, F.J O'Donoghue and M.E. Howard assisted with study conception, design, analysis and preparation of the manuscript.

Conflict of interest: L.M. Hannan received a postgraduate scholarship grant from the National Health and Medical Research Council (Australia), during the conduct of the study. L. Rautela has nothing to disclose. D.J. Berlowitz has nothing to disclose. C.F. McDonald has nothing to disclose. J.M. Cori has nothing to disclose. N. Sheers has nothing to disclose. C. Chao has nothing to disclose. F.J. O'Donoghue has nothing to disclose. M.E. Howard has nothing to disclose.

Support statement: This study was supported by research grants from the Institute for Breathing and Sleep and the Austin Medical Research Foundation. L.M. Hannan received financial support during this project in the form of a postgraduate scholarship from the National Health and Medical Research Foundation (Australia).

\section{References}

1 Hannan LM, Dominelli GS, Chen Y-W, et al. Systematic review of non-invasive positive pressure ventilation for chronic respiratory failure. Respir Med 2014; 108: 229-243.

2 Lloyd-Owen SJ, Donaldson GC, Ambrosino N, et al. Patterns of home mechanical ventilation use in Europe: results from the Eurovent survey. Eur Respir J 2005; 25: 1025-1031.

3 Garner DJ, Berlowitz DJ, Douglas J, et al. Home mechanical ventilation in Australia and New Zealand. Eur Respir J 2013; 41: 39-45.

4 Chu CM, Yu WC, Tam CM, et al. Home mechanical ventilation in Hong Kong. Eur Respir J 2004; 23: 136-141.

5 Hannan LM, Sahi H, Road JD, et al. Care practices and health-related quality of life for individuals receiving assisted ventilation: a cross-national study. Ann Am Thorac Soc 2016; 13: 894-903.

6 Consensus Conference. Clinical indications for noninvasive positive pressure ventilation in chronic respiratory failure due to restrictive lung disease, COPD, and nocturnal hypoventilation - a consensus conference report. Chest 1999; 116: 521-534.

7 Tuggey JM, Elliott MW. Titration of non-invasive positive pressure ventilation in chronic respiratory failure. Respir Med 2006; 100: 1262-1269.

8 Domenech-Clar R, Nauffal-Manssur D, Compte-Torrero L, et al. Adaptation and follow-up to noninvasive home mechanical ventilation: ambulatory versus hospital. Respir Med 2008; 102: 1521-1527.

9 Chatwin M, Nickol AH, Morrell MJ, et al. Randomised trial of inpatient versus outpatient initiation of home mechanical ventilation in patients with nocturnal hypoventilation. Respir Med 2008; 102: 1528-1535.

10 Sheers N, Berlowitz DJ, Rautela L, et al. Improved survival with an ambulatory model of non-invasive ventilation implementation in motor neuron disease. Amyotroph Lateral Scler Front Degener 2014; 15: 180-184.

11 Windisch W. Impact of home mechanical ventilation on health-related quality of life. Eur Respir J 2008; 32: $1328-1336$.

12 Janssens J-P, Borel J-C, Pepin J-L. Nocturnal monitoring of home non-invasive ventilation: the contribution of simple tools such as pulse oximetry, capnography, built-in ventilator software and autonomic markers of sleep fragmentation. Thorax 2011; 66: 438-445.

13 Sassoon CSH, Foster GT. Patient-ventilator asynchrony. Curr Opin Crit Care 2001; 7: 28-33.

14 Chao DC, Scheinhorn DJ, Stearn-Hassenpflug M. Patient-ventilator trigger asynchrony in prolonged mechanical ventilation. Chest 1997; 112: 1592-1599. 
15 Leung P, Jubran A, Tobin MJ. Comparison of assisted ventilator modes on triggering, patient effort, and dyspnea. Am J Respir Crit Care Med 1997; 155: 1940-1948.

16 Vignaux L, Vargas F, Roeseler J, et al. Patient-ventilator asynchrony during non-invasive ventilation for acute respiratory failure: a multicenter study. Intensive Care Med 2009; 35: 840-846.

17 Thille AW, Rodriguez P, Cabello B, et al. Patient-ventilator asynchrony during assisted mechanical ventilation. Intensive Care Med 2006; 32: 1515-1522.

18 Adler D, Perrig S, Takahashi H, et al. Polysomnography in stable COPD under non-invasive ventilation to reduce patient-ventilator asynchrony and morning breathlessness. Sleep Breath 2011; 16: 1081-1090.

19 Atkeson AD, RoyChoudhury A, Harrington-Moroney G, et al. Patient-ventilator asynchrony with nocturnal noninvasive ventilation in ALS. Neurology 2011; 77: 549-555.

20 Fanfulla F, Taurino AE, Lupo ND, et al. Effect of sleep on patient/ventilator asynchrony in patients undergoing chronic non-invasive mechanical ventilation. Respir Med 2007; 101: 1702-1707.

21 Ramsay M, Mandal S, Suh E-S, et al. Parasternal electromyography to determine the relationship between patientventilator asynchrony and nocturnal gas exchange during home mechanical ventilation set-up. Thorax 2015; 70 : 946-952.

22 Fanfulla F, Delmastro M, Berardinelli A, et al. Effects of different ventilator settings on sleep and inspiratory effort in patients with neuromuscular disease. Am J Respir Crit Care Med 2005; 172: 619-624.

23 Crescimanno G, Canino M, Marrone O. Asynchronies and sleep disruption in neuromuscular patients under home noninvasive ventilation. Respir Med 2012; 106: 1478-1485.

24 Carlucci A, Pisani L, Ceriana P, et al. Patient-ventilator asynchronies: may the respiratory mechanics play a role? Crit Care 2013; 17: R54.

25 Berry RB, Chediak A, Brown LK, et al. Best clinical practices for the sleep center adjustment of noninvasive positive pressure ventilation (NPPV) in stable chronic alveolar hypoventilation syndromes. J Clin Sleep Med 2010; 6: 491-509.

26 ACI Respiratory Network. Domiciliary non-invasive ventilation in adult patients: a consensus statement. 2010. www.aci.health.nsw.gov.au/_data/assets/pdf_file/0008/159794/ACI-NIV-guidelines.pdf Date last accessed: March 10, 2019.

27 McKim DA, Road J, Avendano M, et al. Home mechanical ventilation: a Canadian Thoracic Society clinica practice guideline. Can Respir J 2011; 18: 197-215.

28 National Institute for Health and Clinical Excellence. Motor Neurone Disease: The Use of Non-invasive Ventilation in the Management of Motor Neurone Disease. London, NICE, 2010.

29 Johns MW. A new method for measuring daytime sleepiness: the Epworth sleepiness scale. Sleep 1991; 14: 540-545.

30 Åkerstedt T, Gillberg M. Subjective and objective sleepiness in the active individual. Int J Neurosci 1990; 52: 29-37.

31 Buysse DJ, Reynolds CF III, Monk TH, et al. The Pittsburgh Sleep Quality Index: a new instrument for psychiatric practice and research. Psychiatry Res 1989; 28: 193-213.

32 Krupp LB, LaRocca NG, Muir-Nash J, et al. The Fatigue Severity Scale: application to patients with multiple sclerosis and systemic lupus erythematosus. Arch Neurol 1989; 46: 1121-1123.

33 Ghosh D, Rzehak P, Elliot MW, et al. Validation of the English Severe Respiratory Insufficiency Questionnaire. Eur Respir J 2012; 40: 408-415.

34 Richardson J, Kahn MA, Chen G, et al. Population Norms and Australian Profile using the Assessment of Quality of Life (AQoL) 8D Utility Instrument. 2012. www.aqol.com.au/documents/AQoL-8D/researchpaper72.pdf Date last accessed: March 10, 2019.

35 Berlowitz DJ, Spong J, O’Donoghue FJ, et al. Transcutaneous measurement of carbon dioxide tension during extended monitoring: evaluation of accuracy and stability, and an algorithm for correcting calibration drift. Respir Care 2011; 56: 442-448.

36 Vrijsen B, Testelmans D, Belge C, et al. Patient-ventilator asynchrony, leaks and sleep in patients with amyotrophic lateral sclerosis. Amyotroph Lateral Scler Front Degener 2016; 17: 343-350.

37 Iber C, Ancoli-Israel S, Chesson AL, et al. The AASM Manual for the scoring of sleep and associated events: rules, terminology and technical specifications. 1st Ed. Sleep Med 2007; 9: 165-171.

38 Nickol AH, Hart N, Hopkinson NS, et al. Mechanisms of improvement of respiratory failure in patients with restrictive thoracic disease treated with non-invasive ventilation. Thorax 2005; 60: 754-760.

39 Mokhlesi B, Tulaimat A, Evans AT, et al. Impact of adherence with positive airway pressure therapy on hypercapnia in obstructive sleep apnea. J Clin Sleep Med 2006; 2: 57-62.

40 Patout M, Arbane G, Cuvelier A, et al. Polysomnography versus limited respiratory monitoring and nurse-led titration to optimise non-invasive ventilation set-up: a pilot randomised clinical trial. Thorax 2019; 74: 83-86.

41 Butz M, Wollinsky KH, Wiedemuth-Catrinescu U, et al. Longitudinal effects of noninvasive positive-pressure ventilation in patients with amyotrophic lateral sclerosis. Am J Phys Med Rehabil 2003; 82: 597-604.

42 Bourke SC, Tomlinson M, Williams TL, et al. Effects of non-invasive ventilation on survival and quality of life in patients with amyotrophic lateral sclerosis: a randomised controlled trial. Lancet Neurol 2006; 5: 140-147. 